\title{
Lorentz invariance violation in weak interaction: search for effects in beta decay of polarized neutrons
}

\author{
K. Bodek ${ }^{1}$, A. Kozela ${ }^{2}$, G. Ban ${ }^{4}$, A. Bialek ${ }^{2, *}$, P. Gorel ${ }^{4,3,1, \dagger}$, K. Kirch $^{3,7}$, St. Kistryn ${ }^{1}$, \\ M. Kuzniak ${ }^{1,3, \$}$, O. Naviliat-Cuncic ${ }^{4,8}$, N. Severijns ${ }^{5}$, E. Stephan ${ }^{6}$ and J. Zejma ${ }^{1}$ \\ ${ }^{1}$ Institute of Physics, Jagiellonian University, Cracow, Poland \\ ${ }^{2}$ Institute of Nuclear Physics, Polish Academy of Sciences, Cracow, Poland \\ ${ }^{3}$ Paul Scherrer Institute, Villigen, Switzerland \\ ${ }^{4}$ Universite de Caen Basse-Normandie, CNRS/IN2P3-ENSI, LPC, Caen, France \\ ${ }^{5}$ Katholieke Universiteit Leuven, Leuven, Belgium \\ ${ }^{6}$ Institute of Physics, University of Silesia, Katowice, Poland \\ ${ }^{7}$ Swiss Federal Institute of Technology, Zurich, Switzerland \\ ${ }^{8}$ Michigan State University, East-Lansing, MI 48824, USA \\ E-mail: kazimierz.bodek@uj.edu.p]
}

Lorentz Invariance (LI) belongs to the most basic principles underlying our understanding of nature. No compelling evidence for its violation has been found so far but various tests with increasing accuracy are ongoing motivated by the search for new physics. Among the four interactions, electromagnetism imposes the most stringent constraints while very little is known about the weak interaction in this context. Sidereal and daily modulations have been searched for in beta decay of free polarized neutrons. A sample of about $3 \times 10^{8}$ decay electrons distributed over a three month long data taking period were analyzed. Independent upper limits have been deduced for the coupling of the neutron spin and the electron momentum to an external field postulated as an exotic admixture to the weak interaction dominating in neutron decay [四]. Recently, a formalism was developed within the effective field theory approach including a tensor term in the gauge boson propagator which explicitly violates LI [D]. This formalism allows to interpret the investigated sidereal modulations of the neutron beta decay observables from Ref. [四] in terms of the SME parameters [ [], 团]. The resulting upper limits for certain combinations of these parameters were calculated.

X Latin American Symposium on Nuclear Physics and Applications (X LASNPA),

1-6 December 2013

Montevideo, Uruguay

\footnotetext{
*Present address: University of Alberta, Edmonton, Canada.

${ }^{\dagger}$ Present address: University of Alberta, Edmonton, Canada.

†Present address: Department of Physics, Queen’s University, Kingston, Ontario, Canada.
} 


\section{Introduction}

Lorentz Invariance belongs to the most basic principles underlying our understanding of nature. No compelling evidence for its violation has been found so far but various tests with increasing accuracy are ongoing motivated by the search for new physics. Among the four interactions, electromagnetism reveals the most stringent constraints while very little is known about the weak interaction in this context [5]. The early searches for a rotational invariance violation in the forbidden beta decay rate of ${ }^{90} \mathrm{Y},{ }^{137} \mathrm{Cs}$ and ${ }^{99} \mathrm{Tc}$ nuclei revealed null effects at the $10^{-6}$ level[ [6, 四]. Null result at the $10^{-3}$ level was also reported in the first search for sidereal and daily modulations in the distribution of electrons emitted in the decay of free, polarized neutrons [四]. However, that analysis lacks of deeper physical interpretation since no suitable formalism was available at that time. In this paper we express the upper limits for the sidereal modulation of the neutron decay rates derived in Ref. [W] in terms of the Standard Model Extension (SME) parameters.

In the presence of a hypothetical Lorentz Invariance Violating (LIV) background field which couples to the weak interaction, the distribution of electrons from decaying polarized neutrons, registered in a stationary laboratory located on the Earth, becomes space-time and orientation dependent. J. P. Noordmans et al. in Ref. [[] incorporated LIV in the weak interaction by explicit modification of the gauge boson propagator

$$
\left\langle W^{\mu+}(p) W^{v-}(-p)\right\rangle=\frac{-i\left(g^{\mu v}+\chi^{\mu v}\right)}{M_{W}^{2}},
$$

where $g^{\mu v}$ is the usual Minkowski metric and $\chi^{\mu v}$ a general complex tensor that parametrizes the Lorentz violation. With this extension, in the particle rest system, the differential decay rate for polarized neutrons (mixed, allowed transition) reads:

$$
\begin{gathered}
d W=d W^{0}\left\{1-0.21 \chi_{r}^{00}+\left[0.34 \chi_{r}^{0 l}+0.55\left(\chi_{r}^{l 0}+\varepsilon^{l m k} \chi_{i}^{m k}\right)\right] \frac{p^{l}}{E_{e}}\right. \\
\left.+\frac{\langle m\rangle}{j} \hat{\mathrm{I}}^{k}\left[0.43\left(\chi_{r}^{k 0}-\chi_{r}^{0 k}\right)-0.55 \varepsilon^{k l m} \chi_{i}^{l m}-\left(0.12-0.99 \chi_{r}^{00}\right) \frac{p^{k}}{E_{e}}-0.99\left(\chi_{r}^{l k}-\chi_{i}^{s 0} \varepsilon^{k s l}\right) \frac{p^{l}}{E_{e}}\right]\right\} .
\end{gathered}
$$

$d W^{0}$ stays for the differential decay rate within the Standard Model with $g_{A} / g_{V}=-1.27$ coupling constant ratio. $p^{k}$ and $E_{e}$ describe electron momentum and energy, respectively. Latin indices correspond to the space coordinates and $\frac{\langle m\rangle}{j} \hat{\mathrm{I}}^{k}$ describes the neutron polarization.

If the hypothetical background field is of cosmological origin, the neutron decay rate measured in a stationary laboratory on the Earth should reveal a sidereal modulation with the angular frequency $\omega=2 \pi /\left(23^{h} 56^{m} 4.1^{s}\right)$ (provided no conspiracy exists making this field parallel to the Earth rotation axis). In order to account for the Earth rotation, the LIV tensor $\chi^{\mu v}$ should be transformed to the Sun-centered inertial reference frame (denoted by $X^{\mu v}$ ). This is accomplished by using a rotation matrix $R(\zeta, \omega t)$ where $\zeta$ describes the colatitude of the laboratory and $t$ is the measurement time. Corresponding formulas can be found e.g. in Ref. [四].

\section{Experiment}

The original experiment performed at the cold polarized neutron beam facility FUNSPIN [8] at the Paul Scherrer Institute, Villigen, Switzerland, was devoted to measure the transverse polar- 
ization of electrons emitted in neutron decay which is sensitive to exotic admixtures of scalar and tensor terms to the dominating V-A type interaction [Q $[0]$. The electrons were tracked in specially designed multiwire proportional chambers (MWPC) and their energy was measured by plastic scintillator hodoscopes. The angular coverage of the detectors was approximately $(\pi / 4 \leq \theta \leq 3 \pi / 4$, $\pi / 4 \leq \varphi \leq 3 \pi / 4)$ and $(\pi / 4 \leq \theta \leq 3 \pi / 4,5 \pi / 4 \leq \varphi \leq 7 \pi / 4)$, with $\theta$ and $\varphi$ being polar and azimuthal angles. Detailed description of the design, operation and performance of the detection system can be found in Ref. [W]. The acquired experimental data contain about $3 \times 10^{8}$ electrons with fully reconstructed momenta, collected during a period of three months of data taking.

A key feature of the data, allowing for the modulation analysis, is that they were time stamped by a precise external clock in $1 \mathrm{~s}$ intervals. The overall uncertainty of the absolute synchronization to the GMT time is about $2 \mathrm{~s}$.

\section{Data analysis}

The data were split into gross time bins constituting 1/48 of the sidereal or the solar day, respectively. Finally, the measurements performed in the subsequent days were folded together in order to gain statistics. Apart from the expected spin up-down rate asymmetry, time series of the electron rate integrated over the upper $(\mathrm{U})$ and lower $(\mathrm{L})$ hemispheres and for the neutron polarization pointing upwards (+) and downwards (-), described as $W_{U}^{+}, W_{U}^{-}, W_{L}^{+}, W_{L}^{-}$, respectively, reveal both non-statistical variations and periodic drifts as shown in Fig. $\square$. Most of these effects are obviously of instrumental nature. The fluctuations are caused mainly by randomly distributed periods without beam leading to different statistics accumulated in neighboring time bins. The drifts result from natural ambient temperature changes affecting mainly the efficiency of the MWPC and the electronic threshold of the analog trigger channels.

The integrated experimental partial rates depend on the (unknown) detector efficiency function $\rho(\theta, \varphi, t)$ and are affected by at least three instrumental effects: (i) spin flipper related fake modulation of the detector efficiency - parameter $\eta$, (ii) modulation of the polarization itself parameter $\varepsilon$, and (iii) temperature related effect caused by slowly varying efficiencies of upper and lower parts of the detector - parameter $\lambda$. All these effects are not expected to reveal sizeable sidereal modulations due to the background field. Being governed by the electromagnetic interactions such modulations are constrained much more stringently in other experiments. The first two effects were studied in the past in the performance tests of the experimental apparatus [ए], [2] and have been estimated to $\eta \approx 0.0012$ and $\varepsilon \approx 0.005$, respectively. The asymmetry of the average efficiencies of the lower and upper detector parts was calculated directly from the data: $\lambda \approx 0.05$. These instrumental effects were accounted for in the analysis by the following substitutions in the integrated partial rates calculated using $\llbracket 2$ :

$$
\begin{gathered}
\frac{p^{i}}{E_{e}} \rightarrow \beta^{K} \mathscr{F}_{i}^{K}, \quad i=x, y, z, \quad K=U, L, \quad \frac{\langle m\rangle}{j} \hat{\mathrm{I}}^{i} \rightarrow P_{i}, \quad P_{z}=P, \quad P_{x}=P_{y}=0 \\
W_{U}^{0 \pm} \rightarrow W_{U}^{0 \pm}(1+\lambda)(1 \pm \eta), \quad W_{L}^{0 \pm} \rightarrow W_{L}^{0 \pm}(1-\lambda)(1 \pm \eta), \quad P^{ \pm} \rightarrow \pm(1 \pm \varepsilon) P
\end{gathered}
$$

where the kinematical form factors are defined as:

$$
\mathscr{F}_{i}^{K}(t)=\int_{K} d \Omega \hat{p}_{i} \rho / \int_{K} d \Omega \rho, \quad \beta^{K}(t)=\int_{K} d \Omega(p / E) \rho / \int_{K} d \Omega \rho
$$




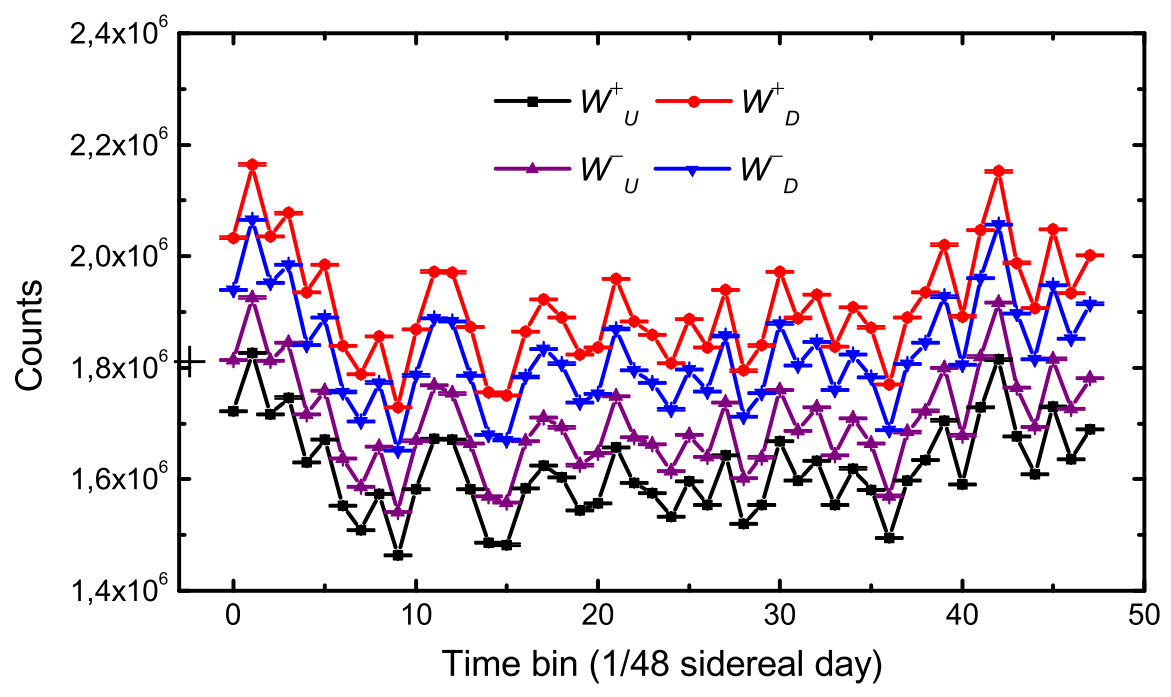

Figure 1: (Color online) Time series of the electron rate integrated over the upper (U) and lower (L) hemispheres and for the neutron polarization pointing upwards (+) and downwards $(-)$, described as $W_{U}^{+}$, $W_{U}^{-}, W_{L}^{+}, W_{L}^{-}$, respectively.

with $i=x, y, z$ and $K=U, L$ corresponding to the counts accumulated in the upper and lower hemispheres, respectively. They can be evaluated with high accuracy approximating the integrals by summing over events.

Two observables constructed from the above defined partial rates appear useful for the extraction of limits of the LIV coefficients $\chi^{\mu v}$ :

$$
\mathscr{E}(t)=\frac{\sqrt{W_{U}^{+} W_{L}^{+}}-\sqrt{W_{U}^{-} W_{L}^{-}}}{\sqrt{W_{U}^{+} W_{L}^{+}}+\sqrt{W_{U}^{-} W_{L}^{-}}}, \quad \mathscr{R}(t)=\frac{\sqrt{W_{U}^{+} W_{U}^{-}}-\sqrt{W_{L}^{+} W_{L}^{-}}}{\sqrt{W_{U}^{+} W_{U}^{-}}+\sqrt{W_{L}^{+} W_{L}^{-}}} .
$$

Since $\chi^{\mu \nu}, \eta, \lambda, \varepsilon$ and the deviations of the kinematical form factors from their mean values are all small parameters, first order Taylor expansion has been used leading to the form:

$$
\begin{aligned}
& \mathscr{E}(t)=-0.01 a_{z}+0.78 b_{0}, \\
& \mathscr{R}(t)=0.05-0.01 a_{x}-0.01 a_{y}+0.28 a_{z}+0.02 b_{0},
\end{aligned}
$$

where

$$
\begin{gathered}
a_{x}=0.55\left(\chi_{r}^{x 0}+\chi_{i}^{y z}-\chi_{i}^{z y}\right), \quad a_{y}=0.55\left(\chi_{r}^{y 0}+\chi_{i}^{z x}-\chi_{i}^{x z}\right), \quad a_{z}=0.55\left(\chi_{r}^{z 0}+\chi_{i}^{x y}-\chi_{i}^{y x}\right), \\
b_{0}=0.43\left(\chi_{r}^{z 0}-\chi_{r}^{0 z}\right)-0.55\left(\chi_{i}^{x y}-\chi_{i}^{y x}\right) .
\end{gathered}
$$

Transforming to the Sun-centered inertial reference frame (colatitude of the Paul Scherrer Institute is equal to $47.52^{\circ}$ ) one arrives at the final expressions for $\mathscr{E}(t)$ and $\mathscr{R}(t)$ :

$$
\begin{aligned}
\mathscr{E}(t) & =-0.23 X_{r}^{T Z}+0.23 X_{r}^{Z T} \\
& +\left[-0.31\left(X_{i}^{Y Z}-X_{i}^{Z Y}\right)-0.25 X_{r}^{T X}+0.25 X_{r}^{X T}\right] \cos (\omega t)
\end{aligned}
$$



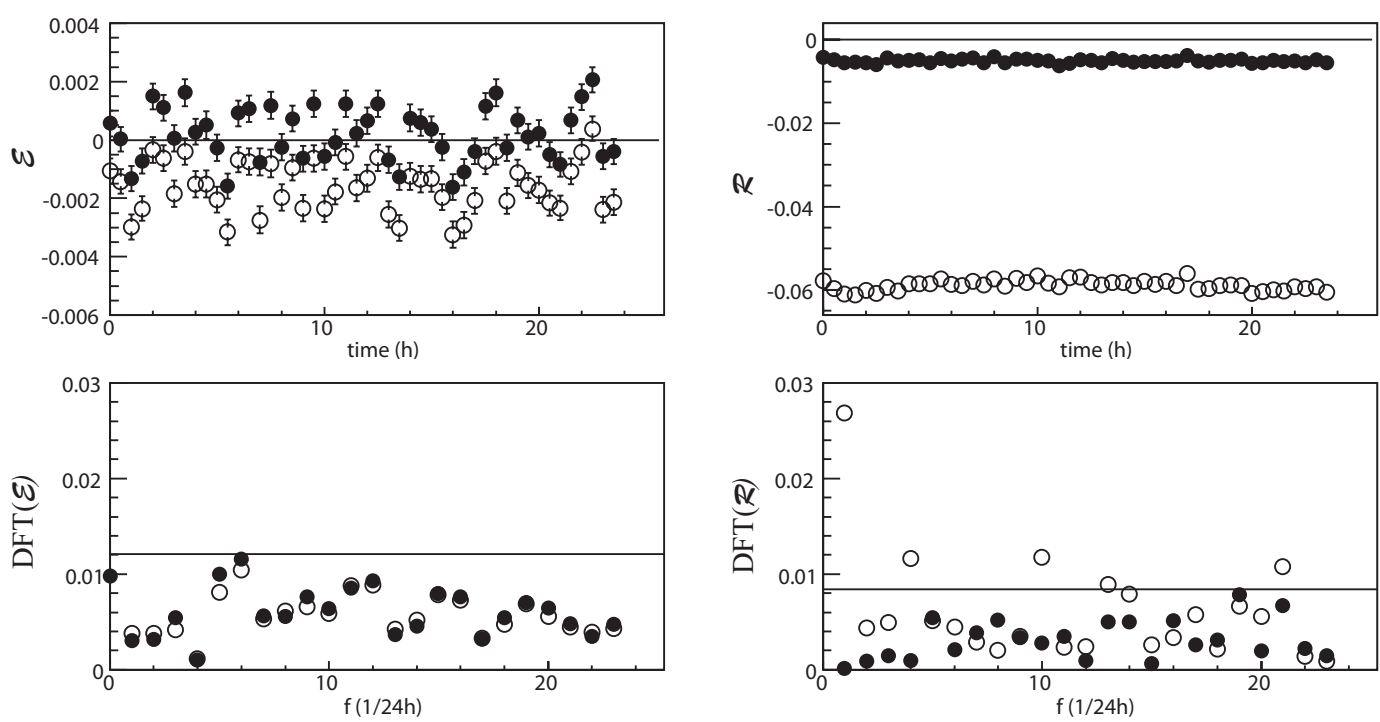

Figure 2: Upper panels: time series for $\mathscr{E}(t)$ (left) and $\mathscr{R}(t)$ (right) raw data (empty symbols) and after correction for instrumental effects (full symbols) folded to one sidereal day. Lower panels: corresponding DFT distributions. In case of a white noise distribution, $95 \%$ of the data points should appear below the horizontal lines.

$$
\begin{aligned}
& +\left[+0.31\left(X_{i}^{X Z}-X_{i}^{Z X}\right)-0.25 X_{r}^{T Y}+0.25 X_{r}^{Y T}\right] \sin (\omega t), \\
\mathscr{R}(t) & =0.05-0.01 X_{r}^{T Z}+0.12 X_{r}^{Z T} \\
& +\left[+0.10\left(X_{i}^{Y Z}-X_{i}^{Z Y}\right)-0.01 X_{r}^{T X}+0.12 X_{r}^{X T}\right] \cos (\omega t) \\
& +\left[-0.10\left(X_{i}^{X Z}-X_{i}^{Z X}\right)-0.01 X_{r}^{T Y}+0.12 X_{r}^{Y T}\right] \sin (\omega t) .
\end{aligned}
$$

Collecting the information from time dependent parts of the above expressions allow one to constrain certain combinations of the LIV coefficients:

$$
\begin{gathered}
\left|+0.62 X_{r}^{X T}-0.28 X_{r}^{T X}\right|<C_{E}+3 C_{R} \\
\left|+0.62 X_{r}^{Y T}-0.28 X_{r}^{T Y}\right|<S_{E}+3 S_{R}
\end{gathered}
$$

where $C_{E}, C_{R}, S_{E}, S_{R}$ are the amplitudes of the cosine and sine terms in $\mathscr{E}(t)$ and $\mathscr{R}(t)$, respectively.

The experimental observables $\mathscr{E}^{\exp }(t)$ and $\mathscr{R}^{\exp }(t)$ obtained from the partial rates are presented in Fig. $\square$ together with their Discrete Fourier Transformants (DFT). Raw and corrected (for the instrumental effects $\eta, \lambda, \varepsilon$ ) observables are shown as open and full dots, respectively.

\section{Results and conclusions}

The confidence levels for the amplitude of the sidereal modulation of $\mathscr{E}^{\exp }(t)$ and $\mathscr{R}^{\exp }(t)$ observables were obtained using the frequentists analysis where the probability distribution of a given signal hypothesis ( $A$ - modulation amplitude, $\omega$-modulation frequency, $\phi$ - modulation phase) is compared with the probability distribution of the null hypothesis $(A=0, \omega, \phi)$. The modulation 

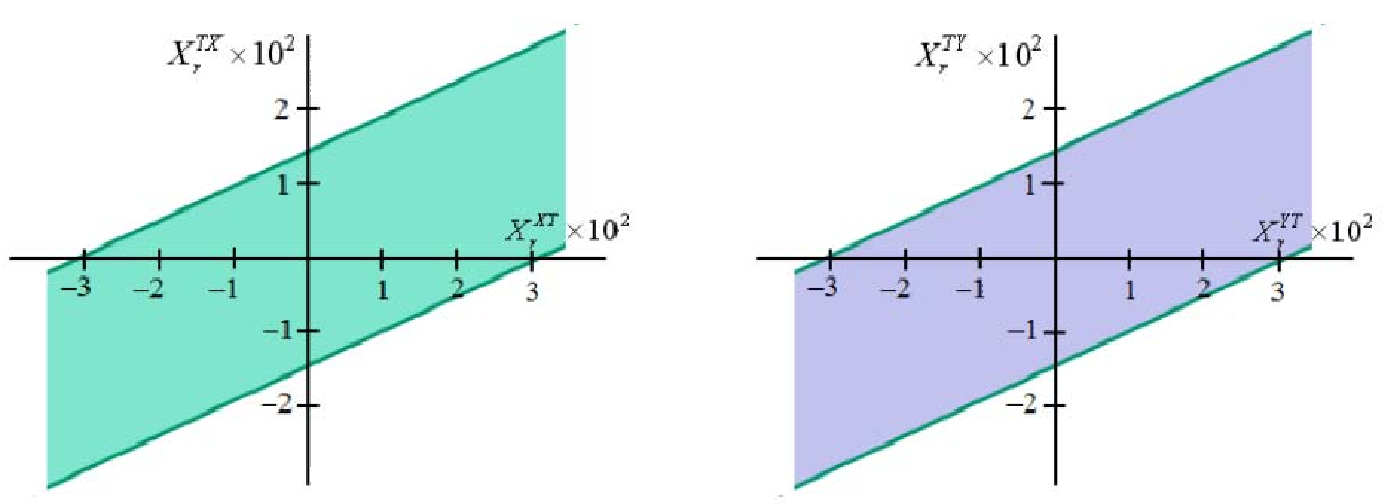

Figure 3: (Color online) Exclusion plots for the SME parameter combinations: $X_{r}^{T X}$ vs. $X_{r}^{X T}$ and $X_{r}^{T Y}$ vs. $X_{r}^{Y T}$. Therein the colored areas represent the allowed parameter values (95\% CL).

phase $\phi$ (sidereal day with respect to the Sun-centered reference frame) remains uncontrolled in our approach due to modest statistics of the data. Finally, we obtain $C_{E}=S_{E}<3.22 \times 10^{-3}$ and $C_{R}=S_{R}<1.89 \times 10^{-3}(95 \% \mathrm{CL})$ leading to

$$
\begin{gathered}
\left|+0.62 X_{r}^{X T}-0.28 X_{r}^{T X}\right|<8.89 \times 10^{-3} \\
\left|+0.62 X_{r}^{Y T}-0.28 X_{r}^{Y X}\right|<8.89 \times 10^{-3}
\end{gathered}
$$

Figure B] shows exclusion plots for the obtained limits. Therein the colored areas represent allowed parameter values. According to our knowledge these are the the first empirical constraints imposed on the two linear combinations of four elements of the Lorentz invariance violation tensor originating from the gauge boson propagator in the weak interaction.

\section{Acknowledgments}

This work was supported in part by the National Science Center, Poland, under grant No. 2012/04/M/ST2/00556. The collaboration is grateful to PSI for excellent support and kind hospitality. Part of the computation work was performed at ACK Cyfronet, Cracow.

\section{References}

[1] A. Kozela et al., CPT and Lorentz Symmetry 5, 174 (2011).

[2] J.P. Noordmans, H.W. Wilschut, R.G.E. Timmermans, Phys. Rev. C 87, 055502 (2013).

[3] D. Colladay and V. A. Kostelecky, Phys. Rev. D 55, 6760 (1997).

[4] D. Colladay and V. A. Kostelecky, Phys. Rev. D 58, 116002 (1998).

[5] V.A. Kostelecky and N. Russell, arXiv:0801.0287v7 (2014).

[6] R. Newman and S. Wisner, Phys. Rev. D 14, 1 (1976).

[7] J.D. Ullman, Phys. Rev. D 17, 1730 (1978). 
[8] K. Bodek et al., Neutron News 11, 29 (2000).

[9] A. Kozela et al., Phys. Rev. Lett. 102, 172301 (2009).

[10] A. Kozela et al., Phys. Rev. C 85, 045501 (2012).

[11] G. Ban et al., Nucl. Instrum. Methods Phys. Res., Sect. 565, 711 (2006).

[12] J. Zejma et al., Nucl. Instrum. Methods Phys. Res. Sect. A 539, 622 (2004). 